PROCEEDINGS OF THE

AMERICAN MATHEMATICAL SOCIETY

Volume 135, Number 7, July 2007, Pages 2193-2203

S 0002-9939(07)08739-4

Article electronically published on March 2, 2007

\title{
A GENERALIZATION \\ OF THE PROLATE SPHEROIDAL WAVE FUNCTIONS
}

AHMED I. ZAYED

(Communicated by Carmen C. Chicone)

\begin{abstract}
Many systems of orthogonal polynomials and functions are bases of a variety of function spaces, such as the Hermite and Laguerre functions which are orthogonal bases of $L^{2}(-\infty, \infty)$ and $L^{2}(0, \infty)$, and the Jacobi polynomials which are an orthogonal basis of a weighted $L^{2}(-1,1)$. The associated Legendre functions, and more generally, the spheroidal wave functions are also an orthogonal basis of $L^{2}(-1,1)$.

The prolate spheroidal wave functions, which are a special case of the spheroidal wave functions, possess a very surprising and unique property. They are an orthogonal basis of both $L^{2}(-1,1)$ and a subspace of $L^{2}(-\infty, \infty)$, known as the Paley-Wiener space of bandlimited functions. They also satisfy a discrete orthogonality relation. No other system of classical orthogonal functions is known to possess this strange property. This raises the question of whether there are other systems possessing this property.

The aim of the article is to answer this question in the affirmative by providing an algorithm to generate such systems and then demonstrating the algorithm by a new example.
\end{abstract}

\section{INTRODUCTION}

Orthogonal polynomials and functions are an important tool in studying function spaces because they provide orthogonal bases for many of these spaces. For example, the Hermite and Laguerre functions, $h_{n}(x)=H_{n}(x) \exp \left(-x^{2} / 2\right)$ and $\mathcal{L}_{n}^{\alpha}(x)=x^{\alpha / 2} \exp (-x / 2) L_{n}(x), n=0,1, \cdots ; \alpha>-1$, are orthogonal bases of $L^{2}(-\infty, \infty)$ and $L^{2}(0, \infty)$, respectively, where $H_{n}(x)$ and $L_{n}(x)$ are the Hermite and Laguerre polynomials, while the Jacobi polynomials $P_{n}^{(\alpha, \beta)}(x), \alpha, \beta>-1$, are an orthogonal basis of $L^{2}(-1,1)$ with respect to the weight function $(1-x)^{\alpha}(1+x)^{\beta}$. The associated Legendre functions, $P_{n}^{m}(x)$, and more generally, the spheroidal wave functions, $P s_{n}^{m}\left(x, \gamma^{2}\right)$, are also an orthogonal basis of $L^{2}(-1,1)$.

The prolate spheroidal wave functions, $P s_{n}^{0}\left(x, \gamma^{2}\right)$, which are a special case of the spheroidal wave functions, possess a very surprising and unique property. They are an orthogonal basis of both $L^{2}(-1,1)$ and a subspace of $L^{2}(-\infty, \infty)$, known as the Paley-Wiener space of bandlimited functions. They also satisfy a discrete orthogonality relation.

Received by the editors October 20, 2005 and, in revised form, March 27, 2006.

2000 Mathematics Subject Classification. Primary 33C47, 44A05; Secondary 42C05, 33C45.

Key words and phrases. Prolate and oblate spheroidal wave functions, orthogonal polynomials and functions, reproducing-kernel Hilbert spaces, bandlimited functions.

(C)2007 American Mathematical Society Reverts to public domain 28 years from publication 
No other system of orthogonal functions is known to possess this strange property. This raises the question of whether other systems of functions possessing this property do exist. The purpose of this article is to answer this question. We shall show that the answer is in the affirmative by providing an algorithm to generate such systems, and then we shall demonstrate the idea by giving a new example.

To explain the mystery surrounding the prolate spheroidal wave functions, it is worth noting that the orthogonality of all the aforementioned systems can be proved using the fact that they are eigenfunctions of Sturm-Liouville boundaryvalue problems on their respective intervals. The same, of course, holds for the orthogonality of the prolate spheroidal wave functions on the interval $(-1,1)$; however, this is not enough to establish their orthogonality on $(-\infty, \infty)$. The latter was proved using the fact that the corresponding Sturm-Liouville differential operator commutes with an integral operator whose eigenfunctions are also the prolate spheroidal wave functions. This commutativity property was described as a lucky accident by Slepian [10] and more recently by Walter [14].

Our approach differs from the classical approach in avoiding the theory of SturmLiouville boundary-value problems all together. Instead, we use the theory of reproducing-kernel Hilbert spaces and compact operators on Hilbert spaces. In fact, we shall show at the end of the article that if one uses the differential operator approach, then the double orthogonality relation of the prolate spheroidal functions is indeed a lucky accident.

The article is organized as follows. In Section 2 we give a brief introduction to the Paley-Wiener space of bandlimited functions and to the prolate spheroidal wave functions. In Section 3 we discuss the theory of reproducing-kernel Hilbert spaces and summarize the main results that will be needed in the sequel. The main result is introduced in Section 4, and in Section 5 we conclude the article with a new example.

\section{Prolate spheroidal Wave Functions}

In this section we give a brief introduction to the prolate spheroidal wave functions and some of their important properties. Most of the material here is collected from different sources [2, 3, 5, 6, 11, 12, 16, see also [1, 4, 15, 17.

Let $B_{\sigma}^{2}$ be the Paley-Wiener space of functions bandlimited to $[-\sigma, \sigma]$, which consists of entire functions of exponential type, with type $\sigma$, that belong to $L^{2}(\mathbb{R})$ when restricted to the real axis. It follows from a theorem, due to Paley and Wiener, that $f \in B_{\sigma}^{2}$ if and only if

$$
f(z)=\frac{1}{\sqrt{2 \pi}} \int_{-\sigma}^{\sigma} F(t) e^{i z t} d t, \text { for some } F \in L^{2}(-\sigma, \sigma) .
$$

The space $B_{\sigma}^{2}$ is a reproducing-kernel Hilbert space so that for any $f \in B_{\sigma}^{2}[18$,

$$
\int_{-\infty}^{\infty} f(x) \frac{\sin \sigma(t-x)}{\pi(t-x)} d x=f(t) .
$$

Now consider the spheroidal differential equation (see [2, 3, 13])

$$
\frac{d}{d x}\left(\left(1-x^{2}\right) \frac{d w}{d x}\right)+\left(\lambda+\gamma^{2}\left(1-x^{2}\right)-\frac{\mu^{2}}{1-x^{2}}\right) w=0,
$$

with real parameters $\lambda, \gamma^{2}$, and $\mu$. For $\mu=m=0,1,2, \ldots$, the spheroidal wave functions, $P s_{n}^{m}\left(x, \gamma^{2}\right)$, are solutions of Eq. (2.2) that are bounded on $(-1,1)$. They 
exist only for special values of $\lambda$, namely, the eigenvalues $\lambda_{n}^{m}\left(\gamma^{2}\right)$. When $\gamma=0$, they reduce to the associated Legendre functions with $\lambda_{n}^{m}(0)=n(n+1)$.

The spheroidal wave functions are orthogonal on $(-1,1)$ and satisfy the relation

$$
\int_{-1}^{1} P s_{n}^{m}\left(x, \gamma^{2}\right) P s_{k}^{m}\left(x, \gamma^{2}\right) d x=\frac{2}{2 n+1} \frac{(n+m) !}{(n-m) !} \delta_{k, n} .
$$

Throughout this article, we shall confine ourselves to the case where $\mu=0$, i.e., we will focus on $P s_{n}^{0}\left(x, \gamma^{2}\right)$ with $\gamma^{2} \neq 0$. If $\gamma^{2}>0, P s_{n}^{0}\left(x, \gamma^{2}\right)$ are called prolate spheroidal wave functions and when $\gamma^{2}<0$, they are called oblate spheroidal wave functions. Let $\gamma=\tau \sigma$, with $\tau, \sigma>0$ and define $\varphi_{n, \sigma, \tau}(t)=\alpha_{n} P s_{n}^{0}\left(t / \tau, \gamma^{2}\right), \quad n=$ $0,1,2, \ldots$, for some constants $\alpha_{n}>0$. It is known that $\varphi_{n, \sigma, \tau}$ satisfy a number of interesting relations, chief among them being the double orthogonality relations

$$
\int_{-\tau}^{\tau} \varphi_{n, \sigma, \tau}(t) \varphi_{m, \sigma, \tau}(t) d t=a_{n} \delta_{m, n}
$$

and

$$
\int_{-\infty}^{\infty} \varphi_{n, \sigma, \tau}(t) \varphi_{m, \sigma, \tau}(t) d t=\delta_{m, n}
$$

They also form an orthogonal basis for $L^{2}(-\tau, \tau)$ and an orthonormal basis for a subspace of $L^{2}(\mathbb{R})$, namely, the space of functions $B_{\sigma}^{2}$ that are bandlimited to $(-\sigma, \sigma)$. It should be noted that one may renormalize $\left\{\varphi_{n, \sigma, \tau}\right\}$ so that they are orthonormal on $L^{2}(-\tau, \tau)$ instead of $L^{2}(\mathbb{R})$, i.e., for some positive constants $\mu_{n}$,

$$
\int_{-\tau}^{\tau} \varphi_{n, \sigma, \tau}(t) \varphi_{m, \sigma, \tau}(t) d t=\delta_{m, n} \quad \text { and } \int_{-\infty}^{\infty} \varphi_{n, \sigma, \tau}(t) \varphi_{m, \sigma, \tau}(t) d t=\mu_{n} \delta_{m, n}
$$

It is also known that the prolate spheroidal wave functions, $\varphi_{n, \sigma, \tau}$, are eigenfunctions of the differential operator

$$
\left(\tau^{2}-t^{2}\right) \frac{d^{2} \varphi_{n, \sigma, \tau}}{d t^{2}}-2 t \frac{d \varphi_{n, \sigma, \tau}}{d t}-\sigma^{2} t^{2} \varphi_{n, \sigma, \tau}=\nu_{n, \sigma, \tau} \varphi_{n, \sigma, \tau},
$$

where $\nu_{n, \sigma, \tau}$ are the eigenvalues, while the oblate spheroidal wave functions, $\psi_{n, \sigma, \tau}$, are eigenfunctions of the differential operator

$$
\left(\tau^{2}-t^{2}\right) \frac{d^{2} \psi_{n, \sigma, \tau}}{d t^{2}}-2 t \frac{d \psi_{n, \sigma, \tau}}{d t}+\sigma^{2} t^{2} \psi_{n, \sigma, \tau}=\tilde{\nu}_{n, \sigma, \tau} \psi_{n, \sigma, \tau} .
$$

The prolate spheroidal wave functions are also eigenfunctions of two integral equations, the first of which is

$$
\int_{-\tau}^{\tau} \varphi_{n, \sigma, \tau}(x) \frac{\sin \sigma(t-x)}{\pi(t-x)} d x=\lambda_{n} \varphi_{n, \sigma, \tau}(t),
$$

where $\lambda_{n}=\lambda_{n, \tau, \sigma}$ are the eigenvalues, and the second is

$$
\int_{-\tau}^{\tau} \varphi_{n, \sigma, \tau}(t) e^{-i \sigma w t / \tau} d t=\gamma_{n, \sigma, \tau} \varphi_{n, \sigma, \tau}(w)
$$

Because the prolate spheroidal wave functions are bandlimited to $(-\sigma, \sigma)$, we have from (2.1),

$$
\int_{-\infty}^{\infty} \varphi_{n, \sigma, \tau}(x) \frac{\sin \sigma(t-x)}{\pi(t-x)} d x=\varphi_{n, \sigma, \tau}(t)
$$


from which we obtain

$$
\frac{\sin \sigma(t-x)}{\pi(t-x)}=\sum_{n=0}^{\infty} \varphi_{n, \sigma, \tau}(t) \varphi_{n, \sigma, \tau}(x),
$$

and also the discrete orthogonality relation

$$
\sum_{n=0}^{\infty} \varphi_{n, \sigma, \tau}(k \pi / \sigma) \varphi_{n, \sigma, \tau}(m \pi / \sigma)=\delta_{k, m}
$$

If the prolate spheroidal wave functions are normalized according to Eq. (2.5), then Eq. (2.11) becomes

$$
\frac{\sin \sigma(t-x)}{\pi(t-x)}=\sum_{n=0}^{\infty} \frac{\varphi_{n, \sigma, \tau}(t) \varphi_{n, \sigma, \tau}(x)}{\mu_{n}} .
$$

The Fourier transform of the prolate spheroidal wave functions satisfies the relations

$$
\int_{-\infty}^{\infty} e^{-i t w} \varphi_{n, \sigma, \tau}(t) d t=(-i)^{n} \sqrt{2 \pi \tau /\left(\sigma \lambda_{n}\right)} \varphi_{n, \sigma, \tau}(\tau w / \sigma) \chi_{\sigma}(w)
$$

and

$$
\int_{-\tau}^{\tau} e^{-i t w} \varphi_{n, \sigma, \tau}(t) d t=(i)^{n} \sqrt{2 \pi \tau \lambda_{n} / \sigma} \varphi_{n, \sigma, \tau}(\tau w / \sigma),
$$

where $\chi_{\sigma}(w)$ is the characteristic function of $(-\sigma, \sigma)$.

To simplify the notation, we normalize the prolate spheroidal wave functions so that they are orthonormal on $(-1,1)$, i.e., we set $\tau=1$ and hence $\gamma=\sigma$ and denote $\varphi_{n, \sigma, 1}$ by $\varphi_{n}$. Hence, from (2.15) we obtain $\int_{-1}^{1} e^{-i t \sigma w} \varphi_{n}(t) d t=\gamma_{n} \varphi_{n}(w)$, where $\gamma_{n}=(i)^{n} \sqrt{2 \pi \tau \lambda_{n} / \sigma}$, which gives the less familiar formula

$$
e^{-i t \sigma w}=\sum_{n} \gamma_{n} \varphi_{n}(w) \varphi_{n}(t), \quad t, w \in[-1,1]
$$

\section{Reproducing-Kernel Hilbert SPACES}

Let $\mathcal{H}$ denote a Hilbert space of functions defined on a set $E$. We denote its inner product and norm by $\langle,\rangle_{\mathcal{H}}$ and $\|-\|_{\mathcal{H}}$, respectively. A function $K(x, y), x, y \in E$ is called a reproducing kernel of $\mathcal{H}$ if: i) for every $y \in E, K(x, y) \in \mathcal{H}$ as a function of $x$; ii) for every $y \in E$ and every $f \in \mathcal{H}, f(y)=\langle f(x), K(x, y)\rangle_{\mathcal{H}}$.

Here it is understood that the inner product applies to functions in $x$. If a reproducing kernel exists, it must be unique. Moreover, the reproducing kernel exists if and only if for every $y \in E$, the point evaluation $f(y)$ is a continuous linear functional on $\mathcal{H}$.

A function $h(x, y)$ is said to be Hermitian if $h(x, y)=\overline{h(y, x)}$ and positive definite if $\sum_{i, j=1}^{n} h\left(y_{i}, y_{j}\right) c_{i} \bar{c}_{j} \geq 0$ for any set of finite points $\left\{y_{i}\right\}_{i=1}^{n}$ and complex numbers $\left\{c_{i}\right\}_{i=1}^{n}$. It is easy to see that if $K(x, y)$ is a reproducing kernel of $\mathcal{H}$, then $K(x, y)$ is Hermitian and positive definite. Conversely, if $K(x, y)$ is a positive definite Hermitian function defined on $E$, then there exists a uniquely determined Hilbert space $\mathcal{H}_{K}$ of functions on $E$ admitting the reproducing kernel $K(x, y)$. If $K(x, y)$ is a reproducing kernel, then the following is true:

$$
K(x, x) \geq 0, K(x, y)=\overline{K(y, x)} \text { and }|K(x, y)|^{2} \leq K(x, x) K(y, y) .
$$


If $K(x, y)$ is a reproducing kernel of $\mathcal{H}$ and $\left\{g_{n}\right\}_{n=0}^{\infty}$ is an orthonormal basis of $\mathcal{H}$, then

$$
K(x, y)=\sum_{n=0}^{\infty} g_{n}(x) \overline{g_{n}(y)}, x, y \in E .
$$

Let $E$ be an arbitrary set and $\mathcal{F}(E)$ be the linear space of all complex-valued functions defined on $E$. Let $\mathcal{H}$ be a Hilbert space with inner product $\langle,\rangle_{\mathcal{H}}$, and $h: E \rightarrow \mathcal{H}$ be a vector-valued function from $E$ into $\mathcal{H}$. Consider the linear mapping $L$ from $\mathcal{H}$ into $\mathcal{F}(E)$ defined by

$$
f(p)=(L F)(p)=\langle F, h(p)\rangle_{\mathcal{H}},
$$

where $L F=f, F \in \mathcal{H}, f \in \mathcal{F}(E)$.

Let $\tilde{\mathcal{H}}$ and $N(L)$ denote the range and the null space of $L$. Let $M=\mathcal{H} \ominus N(L)$, and denote by $P_{M}$ the orthogonal projection from $\mathcal{H}$ into $M$. It has been shown that [8, 9] $\left(\tilde{\mathcal{H}},\langle,\rangle_{\tilde{\mathcal{H}}}\right)$ is a Hilbert space that is isometric to $\left(M,\langle,\rangle_{\mathcal{H}}\right)$, where

$$
\langle f, g\rangle_{\tilde{\mathcal{H}}}=\langle L F, L G\rangle_{\tilde{\mathcal{H}}}=\left\langle P_{M} F, P_{M} G\right\rangle_{\mathcal{H}}
$$

Moreover, $\left(\tilde{\mathcal{H}},\langle,\rangle_{\tilde{\mathcal{H}}}\right)$ is a reproducing-kernel Hilbert space. Now let

$$
K(p, q)=\langle h(p), h(q)\rangle_{\mathcal{H}}, \quad p, q \in E .
$$

Because for any complex numbers $\left\{\alpha_{i}\right\}_{i=1}^{n}$,

$$
\begin{gathered}
0 \leq\left\langle\sum_{i=1}^{n} \alpha_{i} h\left(p_{i}\right), \sum_{j=1}^{n} \alpha_{j} h\left(p_{j}\right)\right\rangle_{\mathcal{H}}=\sum_{i, j=1}^{n} \alpha_{i} \bar{\alpha}_{j}\left\langle h\left(p_{i}\right), h\left(p_{j}\right)\right\rangle_{\mathcal{H}} \\
=\sum_{i, j=1}^{n} \alpha_{i}, \bar{\alpha}_{j} K\left(p_{i}, p_{j}\right),
\end{gathered}
$$

it follows that $K(p, q)$ is positive definite and Hermitian, and hence it is the reproducing kernel of some reproducing-kernel Hilbert space (possibly finite-dimensional space). In fact, that space is exactly $\tilde{\mathcal{H}}$.

We have

$$
\|f\|_{\tilde{\mathcal{H}}}=\inf _{F}\|F\|_{\mathcal{H}} \leq\|F\|_{\mathcal{H}}
$$

where the infimum is taken over all $F$ such that $L F=f$. Furthermore, $L$ is an isometry between $\mathcal{H}$ and $\tilde{\mathcal{H}}$ if and only if $\{h(p): p \in E\}$ is complete in $\mathcal{H}$ and in this case $N(L)=\{0\}$ and hence

$$
\|f\|_{\tilde{\mathcal{H}}}=\left\|P_{M} F\right\|_{\mathcal{H}}=\|F\|_{\mathcal{H}}
$$

Now we apply these general results to a specific case. Let $d \mu$ be a $\sigma$-finite positive measure and $T$ be a $d \mu$-measurable set. Consider the Hilbert Space $\mathcal{H}=L^{2}(T, d \mu)$ consisting of all complex-valued functions $F$ such that

$$
\|F\|_{L^{2}(T, d \mu)}^{2}=\int_{T}|F(t)|^{2} d \mu(t)<\infty .
$$

Let $E$ be an arbitrary set and $h(t, p)$ be a fixed complex-valued function on $T \times E$, such that

$$
h(t, p) \in L^{2}(T, d \mu) \text { for any } p \in E \text {. }
$$


Let $L$ be the linear mapping $L: L^{2}(T, d \mu) \rightarrow \mathcal{F}(E)$ defined by

$$
f(p)=(L F)(p)=\int_{T} F(t) \bar{h}(t, p) d \mu(t), \quad F \in L^{2}(T, d \mu) .
$$

Then, the set of all such $f$ 's is a reproducing-kernel Hilbert space $\tilde{\mathcal{H}}=\mathcal{H}_{K}$ with reproducing kernel

$$
K(p, q)=\int_{T} h(t, q) \overline{h(t, p)} d \mu(t)
$$

with $f(q)=\langle f, K(., q)\rangle_{\tilde{\mathcal{H}}}$ and

$$
\|f\|_{\mathcal{H}_{K}}^{2}=\inf \int_{T}|F(t)|^{2} d \mu(t) \leq \int_{T}|F(t)|^{2} d \mu(t)
$$

where the infimum is taken over all $F$ such that $L F=f$. Moreover, the integral transform (3.7) is an isometry between $L^{2}(T, d \mu)$ and $\mathcal{H}_{K}$ if and only if $\{h(t, p)\}_{p \in E}$ is complete in $L^{2}(T, d \mu)$.

\section{THE MAIN RESULT}

Now we state and prove our main result.

Theorem 4.1. Let $T$ be an interval of the form $[a, b]$, where $-\infty<a<b<\infty$, and $E$ be a measurable subset of $\mathbb{R}$ containing $T$, i.e., $T \subset E$. Let $h(t, p)$ be real and continuous on $T \times E$, and assume that $\{h(t, p)\}_{p \in E}$ is complete in $L^{2}(T, d \mu)$. Then there exist a reproducing-kernel Hilbert space, $\tilde{\mathcal{H}}$, comprising functions defined on $E$ and an orthonormal basis $\left\{\varphi_{n}\right\}_{n=0}^{\infty}$ of $L^{2}(T, d \mu)$ with the property that $\varphi_{n}$ can be naturally extended to functions $\Phi_{n}$ defined on $E$ such that $\Phi_{n} \in \tilde{\mathcal{H}}$ and

i)

$$
\int_{T} \Phi_{m}(t) \Phi_{n}(t) d \mu(t)=\int_{T} \varphi_{m}(t) \varphi_{n}(t) d \mu(t)=\delta_{m, n}
$$

ii)

$$
\left\langle\Phi_{m}, \Phi_{n}\right\rangle_{\tilde{\mathcal{H}}}=\mu_{n} \delta_{m, n}, \text { with } \mu_{n}=1 /|\lambda|_{n}^{2}
$$

where $\left\{\lambda_{n}\right\}$ are the eigenvalues of a compact operator on $L^{2}(T, d \mu)$;

iii) $\left\{\varphi_{n}\right\}_{n=0}^{\infty}$ are solutions of the Fredholm integral equation of the second kind

$$
\mu_{n} \int_{a}^{b} \varphi_{n}(x) K(x, p) d \mu(x)=\varphi_{n}(p), \quad p \in T
$$

iv)

where $K(x, p)$ is the reproducing kernel of $\tilde{\mathcal{H}}$;

$$
h(t, p)=\sum_{n=0}^{\infty} \lambda_{n} \varphi_{n}(t) \varphi_{n}(p), \quad t, p \in T
$$

v)

$$
K(p, q)=\sum_{n=0}^{\infty} \frac{\Phi_{n}(p) \Phi_{n}(q)}{\mu_{n}}
$$


vi) in addition, if there exists a sequence of points $\left\{p_{n}\right\}_{n=0}^{\infty} \subset E$ such that $\left\{h\left(t, p_{n}\right)\right\}$ is an orthonormal basis of $L^{2}(T, d \mu)$, then for any $f \in \tilde{\mathcal{H}}$,

$$
f(p)=\sum_{n=0}^{\infty} f\left(p_{n}\right) K\left(p, p_{n}\right)
$$

and

$$
\sum_{n=0}^{\infty} \frac{\Phi_{n}\left(p_{k}\right) \Phi_{n}\left(p_{m}\right)}{\mu_{n}}=\delta_{k, m}
$$

Proof. i) Define

$$
f(p)=(L F)(p)=\int_{a}^{b} F(t) h(t, p) d \mu(t), \quad p \in E
$$

for all $F \in L^{2}(T, d \mu)=\mathcal{H}$. Since $h(t, p)$ is continuous on $T \times E$, it is in $L^{2}(T, d \mu)$ for any fixed $p \in E$; hence, $(L F)(p)$ is well defined for any $p \in E$. Moreover, we have

$$
|(L F)(p)|^{2} \leq\left(\int_{a}^{b}|F(t)|^{2} d \mu(t)\right)\left(\int_{a}^{b}|h(t, p)|^{2} d \mu(t)\right) .
$$

Since $h(t, p)$ is continuous on the closed square $Q=T \times T$, we have

$$
\int_{a}^{b}|(L F)(p)|^{2} d \mu(p) \leq\|F\|_{\mathcal{H}}^{2} \iint_{Q}|h(t, p)|^{2} d \mu(t) d \mu(p)<\infty,
$$

which implies that when $L F(p)$ is restricted to $T$, it is in $L^{2}(T, d \mu)$, i.e., $L$ is a continuous linear transformation on $L^{2}(T, d \mu)$. Because $h(t, p)$ is real, it is easy to see that $L$ is self-adjoint. Moreover, by standard arguments one can show that $L$ is compact.

Since $L$ is a self-adjoint, compact transformation on $L^{2}(T, d \mu)=\mathcal{H}$, it has a sequence of eigenvalues $\left\{\lambda_{n}\right\}_{n=1}^{\infty}$ and eigenfunctions $\left\{\varphi_{n}\right\}_{n=1}^{\infty}$ such that $L \varphi_{n}=$ $\lambda_{n} \varphi_{n}$ for all $n$, i.e.,

$$
\int_{a}^{b} \varphi_{n}(t) h(t, p) d \mu(t)=\left\langle\varphi_{n}(\cdot), h(., p)\right\rangle_{\mathcal{H}}=\lambda_{n} \varphi_{n}(p) .
$$

If $\left\{\tilde{\varphi}_{k}\right\}$ is an orthogonal basis of the null space of $L$, then the set $\left\{\tilde{\varphi}_{k}, \varphi_{k}\right\}$ is an orthogonal basis of $L^{2}(T, d \mu)$. But from the assumption that $\{h(t, p)\}_{p \in E}$ is complete in $L^{2}(T, d \mu)$, it follows that the null space of $L$ is $\{0\}$, and hence the eigenfunctions $\left\{\varphi_{n}\right\}$ are an orthogonal basis of $L^{2}(T, d \mu)$. From now on we shall normalize them so that $\left\langle\varphi_{m}, \varphi_{n}\right\rangle_{\mathcal{H}}=\delta_{m, n}$, and hence, they form an orthonormal basis of $L^{2}(T, d \mu)$.

But from (3.7) and the discussion following it, it follows that $L$ also maps $\mathcal{H}=$ $L^{2}(T, d \mu)$ into a vector subspace, $\tilde{\mathcal{H}}$, of the space, $\mathcal{F}(E)$, of all functions defined on $E$. The space $\tilde{\mathcal{H}}$, which consists of all functions of the form (3.7), is a reproducingkernel Hilbert space with inner product given by (3.3) and a reproducing kernel given by (3.8).

Let us denote the image of $\varphi_{n}(t)$ under $L$ by $\Phi_{n}(p), p \in E$. Evidently, $\Phi_{n}(p)=$ $\lambda_{n} \varphi_{n}(t)$ for $p \in T$, and as a result, we may conclude that $\Phi_{n}(p)$ is an extension of $\varphi_{n}(t)$ from $T$ into $E$. 
ii) From (3.3) we have

$$
\left\langle\Phi_{m}, \Phi_{n}\right\rangle_{\tilde{\mathcal{H}}}=\left\langle\lambda_{m} \varphi_{m}, \lambda_{n} \varphi_{n}\right\rangle_{\tilde{\mathcal{H}}}=\left\langle P_{M} \varphi_{m}, P_{M} \varphi_{n}\right\rangle_{\mathcal{H}} .
$$

But since $\{h(t, p)\}_{p \in E}$ is complete, we have

$$
\lambda_{m} \lambda_{n}\left\langle\varphi_{m}, \varphi_{n}\right\rangle_{\tilde{\mathcal{H}}}=\left\langle\varphi_{m}, \varphi_{n}\right\rangle_{\mathcal{H}}=\delta_{m, n}
$$

which implies

$$
\left\|\varphi_{n}\right\|_{\tilde{\mathcal{H}}}^{2}=\mu_{n}, \text { where } \mu_{n}=\frac{1}{\left|\lambda_{n}\right|^{2}} .
$$

iii) We also have from (4.5),

$$
\begin{aligned}
\lambda_{n} \int_{a}^{b} \varphi_{n}(t) h(t, p) d \mu(t) & =\int_{a}^{b} h(t, p) d \mu(t)\left\{\int_{a}^{b} \varphi_{n}(x) h(x, t) d \mu(x)\right\} \\
& =\int_{a}^{b} \varphi_{n}(x)\left\{\int_{a}^{b} h(t, p) h(x, t) d \mu(t)\right\} d \mu(x) \\
& =\int_{a}^{b} \varphi_{n}(x) K(x, p) d \mu(x) .
\end{aligned}
$$

But once more from (4.5), we have

$$
\lambda_{n}^{2} \varphi_{n}(p)=\lambda_{n} \int_{a}^{b} \varphi_{n}(t) h(t, p) d \mu(t),
$$

which, when combined with (4.9), leads to the integral equation

$$
\int_{a}^{b} \varphi_{n}(x) K(x, p) d \mu(x)=\lambda_{n}^{2} \varphi_{n}(p)=\frac{1}{\mu_{n}} \varphi_{n}(p),
$$

which is equivalent to (4.1).

iv) This follows from (4.5) and the fact that $\left\{\varphi_{n}\right\}$ is an orthonormal basis for $L^{2}(T, d \mu)$.

v) This follows from (3.1) and the fact that $\left\{\varphi_{n}(x) / \sqrt{\mu_{n}}\right\}$ is an orthonormal basis of $\tilde{\mathcal{H}}$.

v) Let us denote $h\left(t, p_{n}\right)$ by $h_{n}(t)$. Since $\left\{h_{n}(t)\right\}$ is an orthonormal basis of $L^{2}(T, d \mu)$, we have $F(x)=\sum_{n=0}^{\infty}\left\langle F, h_{n}\right\rangle h_{n}(x)$. By applying the transformation $L$ to this relation and noting that $f\left(p_{n}\right)=\left\langle F, h_{n}\right\rangle$, we obtain (4.2).

Finally, because $\left\{h_{n}(t)\right\}$ is orthonormal, it follows that $K\left(p_{m}, p_{n}\right)=\delta_{m, n}$, and hence (4.3) follows from (iv).

Remarks. 1) Clearly, (i), (ii), and (iii) generalize (2.5) and (2.8), while (iv), (v) and (4.3) generalize (2.16), (2.13) and (2.12).

2) Theorem 4.1 is essentially an existence theorem. Finding explicit examples is not easy because it depends on finding explicit solutions of integral equations.

3) Since finding the eigenvalues and eigenfunctions of boundary-value problems involving differential operators is easier than finding those of integral equations, one may be tempted to derive the double orthogonality property by first starting with a boundary-value problem and then converting it into an integral equation. This approach is bound to fail because in this case the kernel of the integral equation, which is the Green's function of the problem $G(x, t)$, does not satisfy the hypotheses of Theorem 4.1, the reason being that the Green's function is generally given by two expressions, one for $a \leq x \leq t$ and one for $t \leq x \leq b$ and therefore, it does not 
have a natural extension for either $x$ or $t$ beyond the original interval of definition $[a, b]$.

\section{EXAMPLES}

First, we show that the prolate spheroidal wave functions are a special case of Theorem 4.1 .

Example 5.1. First, let us observe that if $f \in B_{\sigma}^{2}$, then $f$ can be written in the form $f(t)=\int_{-1}^{1} e^{i \sigma x t} F(x) d x$, for some $F \in L^{2}(-1,1)$. This may suggest that we take the function $h(x, t)$ in the theorem to be $e^{i \sigma x t}$; however this function does not satisfy the hypotheses of the theorem. Therefore, we will first take $h(x, t)=\cos \sigma x t$, and consider the integral operator

$$
\left(K_{1} F\right)(t)=\int_{-1}^{1} F(x) \cos (\sigma x t) d x=f(t),
$$

acting on the space $L_{\text {even }}^{2}(T)$, of all even functions in $L^{2}(T)$, where $T=[-1,1]$. That $h(x, t)$ is complete follows from the Riemann-Lebesgue lemma.

Because $\varphi_{n}$ is real and even when $n$ is even, it follows from (2.9) that the eigenfunctions of the integral equation

$$
\int_{-1}^{1} \phi(x) \cos (\sigma x t) d x=\lambda \phi(t)
$$

are $\left\{\varphi_{2 n}(x)\right\}$ with eigenvalues $\gamma_{2 n}$. Hence, the extension of $\left\{\varphi_{2 n}(x)\right\}$ to the whole real line is an orthogonal basis for the space of all even bandlimited functions.

Secondly, we take $h(x, t)=\sin \sigma x t$, and consider the integral operator

$$
\left(K_{2} F\right)(t)=\int_{-1}^{1} F(x) \sin (\sigma x t) d x=f(t),
$$

acting on the space $L_{\text {odd }}^{2}(T)$. By the same reasoning, we prove that the extension of $\left\{\varphi_{2 n+1}(x)\right\}$ to the whole real line is an orthogonal basis for the space of all odd bandlimited functions. The result now follows since $L^{2}(T)=L_{\text {even }}^{2}(T) \oplus L_{\text {odd }}^{2}(T)$.

Example 5.2. Let $h(x, t)=e^{\gamma x t,}, \gamma \neq 0$ and consider the operators $K$ defined by

$$
(K F)(t)=\int_{-1}^{1} e^{\gamma t x} F(x) d x=f(t) .
$$

Although one can use standard methods for solving integral equations to find the eigenfunctions of this operator, we will use an easier, but indirect method, to do so. Now consider the differential operator $L_{x}=\frac{d}{d x}\left(1-x^{2}\right) \frac{d}{d x}+\gamma^{2} x^{2}$. It is easy to see that $L_{t}(K F)(t)=\left(1-t^{2}\right) f^{\prime \prime}-2 t f^{\prime}+\gamma^{2} t^{2} f$, or

$$
L_{t}(K F)(t)=\int_{-1}^{1} e^{\gamma t x} F(x)\left\{\gamma^{2} x^{2}\left(1-t^{2}\right)-2 \gamma t x+\gamma^{2} t^{2}\right\} d x .
$$

On the other hand, we also have

$$
K\left(L_{x} F\right)(t)=\int_{-1}^{1} e^{\gamma t x}\left\{\frac{d}{d x}\left[\left(1-x^{2}\right) F^{\prime}(x)\right]+\gamma^{2} x^{2} F(x)\right\} d x=I_{1}+I_{2},
$$

where

$$
I_{1}=\int_{-1}^{1} e^{\gamma t x}\left\{\frac{d}{d x}\left(1-x^{2}\right) F^{\prime}(x)\right\} d x \text { and } \quad I_{2}=\gamma^{2} \int e^{\gamma t x} x^{2} F(x) d x .
$$


By integrating $I_{1}$ by parts twice, we obtain

$$
I_{1}=\gamma t \int_{-1}^{1} F(x) \frac{d}{d x}\left\{\left(1-x^{2}\right) e^{\gamma t x}\right\} d x=\gamma t \int_{-1}^{1} e^{\gamma t x} F(x)\left\{\gamma t\left(1-x^{2}\right)-2 x\right\} d x .
$$

Thus, by adding $I_{1}$ and $I_{2}$ we have

$$
K\left(L_{x} F\right)(t)=\int_{-1}^{1} e^{\gamma t x} F(x)\left\{\gamma^{2} t^{2}\left(1-x^{2}\right)-2 \gamma t x+\gamma^{2} x^{2}\right\} d x .
$$

By comparing (5.2) and (5.3), we conclude that $K$ and $L$ commute; hence, they have the same eigenfunctions. But from (2.7), we conclude that the oblate spheroidal wave functions are the eigenfunctions of $K$.

It is easy to see that the space $\left(\tilde{\mathcal{H}},\langle,\rangle_{\tilde{\mathcal{H}}}\right)$ consists of all functions $f(t)$ of the form (5.1) and the inner product is given by [7, p. 62]

$$
\langle f, g\rangle_{\tilde{\mathcal{H}}}=\frac{1}{2 \pi} \int_{-\infty}^{\infty} f(i t) \overline{g(i t)} d t .
$$

The reproducing kernel is given by $K_{\gamma}(t, \eta)=\sinh \gamma(t-\eta) / \pi(t-\eta)$. It follows that the oblate spheroidal wave functions are orthogonal on $(-1,1)$ and also on the imaginary axis. Moreover, they are an orthogonal basis of $L^{2}(-1,1)$ and of the Hilbert space $\tilde{\mathcal{H}}$ because the exponential functions are complete.

\section{REFERENCES}

[1] J. Boyd, Approximation of an analytic function on a finite interval by a bandlimited function and conjectures on properties of prolate spheroidal functions, Appl. Comput. Harmon. Anal., Vol. (2003), 168-176. MR2007058 (2004g:41027)

[2] A. Erdelyi, W. Magnus, F. Oberhettinger, F. Tricomi, Higher Transcendental Functions, Vol. III, Bateman Manuscript, McGraw-Hill, New York (1955). MR0066496 (16:586c)

[3] C. Flammer, Spheroidal Wave Functions, Stanford University Press, California (1957). MR0089520 (19:689a)

[4] K. Khare and N. George, Sampling theory approach to prolate spheroidal wavefunctions, J. Phys. Ser. A, Vol. 36(2003), 10011-10021. MR2024509 (2004m:33029)

[5] H. Landau and H. O. Pollak, Prolate spheroidal wave functions, Fourier analysis and uncertainty-III, Bell Syst. Tech. J. Vol. 41 (1962), pp. 1295 - 1336. MR0147686 (26:5200)

[6] H. Landau and H. O. Pollak, Prolate spheroidal wave functions, Fourier analysis and uncertainty-II, Bell Syst. Tech. J. Vol. 40 (1961), pp. 65 - 84. MR0140733 (25:4147)

[7] S. Saitoh, Integral Transforms, Reproducing Kernels and Their Applications, Pitman Research Notes in Math., Vol. 369 (1997), Addison Wesley Longman, United Kingdom. MR.1478165 (98k:46041)

[8] S. Saitoh, The Theory of Reproducing Kernels and Its Applications, Pitman Research Notes in Math., Vol. 189 (1988), Longman Scientific \& Technical, United Kingdom. MR0983117 (90f:46045)

[9] S. Saitoh, Hilbert spaces induced by Hilbert space valued functions, Proc. Amer. Math. Soc., Vol. 89 (1983), pp. 74 -78. MR0706514 (84h:44001)

[10] D. Slepian, Some comments on Fourier analysis, uncertainty and modeling, SIAM Rev., Vol. 25, (1983), pp. 379 - 393. MR0710468 (84i:94016)

[11] D. Slepian, Prolate spheroidal wave functions, Fourier analysis and uncertainty-IV: Extensions to many dimensions; Generalized prolate spheroidal functions , Bell Syst. Tech. J. Vol. 43 (1964), pp. 3009 -3058. MR0181766 (31:5993)

[12] D. Slepian and H. O. Pollak, Prolate spheroidal wave functions, Fourier analysis and uncertainty-I, Bell Syst. Tech. J. Vol. 40 (1961), pp. 43 -64. MR0140732 (25:4146)

[13] H. Volkmer, Spheroidal Wave Functions, in Handbook of Mathematical Functions, Nat. Bureau of Stds., Applied Math. Series 2004. 
[14] G. Walter, Differential operators which commute with characteristic functions with applications to a lucky accident, J. Complex Variables, Vol. 18 (1992), 7-12. MR.1157019 (93a:34101)

[15] G. Walter and X. Shen, Wavelets based on prolate spheroidal wave functions, J. Fourier Anal. Appls., Vol. 10 (2004), 1-26. MR2045522 (2005c:42041)

[16] G. Walter and X. Shen, Sampling with prolate spheroidal wave functions, J. Sampl. Theory: Signal \& Image Process., Vol. 2 (2003), 25-52. MR2002855 (2004k:42064)

[17] H. Xiao, V. Rokhlin, and N. Yarvin, Prolate spheroidal wavefunctions, quadrature and interpolation, J. Inverse Problems, Vol. 17 (2001), 805-838. MR.1861483 (2002h:41049)

[18] A. I. Zayed, Advances in Shannon's Sampling Theory, CRC Press, Boca Raton, Fl (1993). MR:1270907 (95f:94008)

Department of Mathematical Sciences, DePaul University, Chicago, Illiniois 60614

E-mail address: azayed@condor.depaul.edu 